\title{
The analysis of Grooming Factor on Optimization Algorithms in
}

\section{All-optical Network}

\author{
Su-xia Cui ${ }^{1}$, Yuan Yuan ${ }^{2}$ \\ ${ }^{1}$ Binzhou Polytechnic, Binzhou, Shandong, China, 256600 \\ ${ }^{2}$ Binzhou Polytechnic, Binzhou, Shandong, China, 256600
}

\begin{abstract}
The most important thing in all optical networks is to reduce the cost of using the network in electronic processing equipment, along with wavelength division multiplexing (WDM) technology are increasingly improved. Add-drop multiplexer (ADM) is not only the most important equipment but also the most basic equipment using the point difference in all-optical networks. The function of the ADM processing device, is a separation or insert the desired wavelength to networks. The key to reducing the cost is to reduce the number of ADM uses and minimize the number of ADMs. Traffic grooming problem is an important generalization of the ADM minimization problem, which is NP-hard. The specific way is to effectively package the number of available wavelengths ADMS on low speed traffic streams by requiring the number of wavelength per fiber and the required network, so as to reduce the network cost as much as possible. Synchronous optical network (SONET), which is a fiber-optic network for high-speed data communications transmission network .This paper mainly use the algorithm GROOMBYSC(k) to solve the traffic grooming problems in bi-directional interconnected SONET/WDM rings networks. The algorithm analysis shows that the algorithm has a better approximation ratio. We further improve the algorithm by computational analysis. We obtain a similar result by using the algorithm, and the approximation ratio is $2 \ln g+o(\ln g)$, where $g$ is the grooming factor. Algorithm analysis shows that the grooming factor smaller than its approximation ratio of the approximation algorithm, the better the performance of the algorithm can be seen, what's more we can obtain proved a general of the approximation algorithm.
\end{abstract}

Keywords: Bidirectional SONET ring; Grooming factor; Graph coloring problem; Approximation ratio;

\section{Introduction}

With the rapid development of modern network technology, the demand for capacity and quality of service communication networks become the driving force of the growing network technology research. In order to overcome by the speed signal processing to achieve information exchange network, each node brings electronic bottleneck, it made the most promising technologies, the all-optical network. The so-called all-optical network is the network from the source node to the destination node of data transmission and information exchange between the whole processes is carried out in the optical domain, i.e., the end of the complete optical path, the intermediate electrical signal without any intervention, and the main medium of the transmission is an optical fiber.

WDM network is a cross- switching nodes are connected by a fiber link composed, A WDM wavelength channels carrying both low- speed flow is also able to carry traffic. In a communication system, most of the connection has a small bandwidth request, such as voice or text data, etc. Under no circumstances traffic grooming, each connection will occupy a separate wavelength channel. In 
WDM networks, the wavelength is a limited resource, so often there is not enough wavelengths to each connection provides for an independent wavelength channels. Therefore, it is very necessary for low-bandwidth connections to allow multiple requests to share the same wavelength channel. Traffic grooming traffic is converged to a plurality of connection requests technical single wavelength channel, which greatly increases the bandwidth utilization rate of the wavelength channel, for example, if a wavelength channel bandwidth is OC-48, a bandwidth request is a linking group OC-12 (i.e., 0.622Gbit / s), then the connection may be to divert the four wavelength channels to an OC-48, wherein, to ease factor is 4.

In all-optical networks, basic electronic processing equipment using Add-Drop Multiplexer (ADM), whose functions is to separate the network or insert the desired wavelength, that is, the optical network temporarily unneeded wavelengths from the fiber separated, the wavelength of the network need to insert a channel in a data network for transmission of information.

In fact, the traffic grooming problem is converge the low-speed to high-capacity optical path of transmission traffic through effective multiplexing and switching processing technology, which is to improve the utilization of network resources. Using graph theory terms, traffic grooming problem can be seen as a path coloring problem, so most of which share an edge path $g$ ( $g$ is the grooming factor). From the perspective of ADM, each optical path use two ADMs, each endpoint requires one ADM. At a node case by the same edge with $g$ optical paths having the same wavelength to arrive one node, and they may use the same ADM, thus it can save g-1 ADMs, its purpose is to minimize the use of the number of ADM ${ }^{[1]}$.

With the maturity of the rapid development of modern communication networks and optical fiber communication technology, the mid -second century, the United States proposed a fiber -based network communication technology, synchronous optical network (SONET), which is a fiber-optic network for high-speed data communications transmission network, compared with the general network, SONET spread further example, the most important feature of the SONET network is support the ring topology structure network .

A SONET ring is composed of two or more nodes of a SONET/WDM closure ring, which is part of the fiber optic working ring, as part of the protection ring. Working ring is used for data transmission network need. Protection ring is a spare ring. SONET/WDM ring has a self-test function, when the working ring failure in the data transmission process, SONET/WDM ring will self-test and within a very short time the protection ring provides control information to the protection ring is in working condition. Therefore, SONET / WDM ring is also known as self-healing ring, and it has the function of self-healing capabilities. SONET / WDM ring is divided into two kinds of unidirectional and bidirectional ring. In unidirectional SONET / WDM ring, the direction of the working ring is the clockwise direction, i.e., in the data transmission is clockwise direction when carried out traffic transmission in SONET/WDM ring. In the case of bidirectional SONET / WDM ring, the direction of the working ring is bi-directional, i.e., both clockwise and counterclockwise directions opposite, when the two nodes adjacent is traffic transmission using two opposite clockwise and counterclockwise direction to data transmission . ${ }^{[2]}$

In electronic communications networks, SONET / WDM ring is widely used. Traditional SONET ring using a single wavelength, however, with the advent of WDM technology, in order to meet the growing traffic demands, these rings need to be upgraded to WDM, and multiple SONET ring cross- connected to provide a large area on the convergence.

In a cross-connected SONET / WDM ring, there are two kinds of node, that is, non-cross connections node and cross- connections node. A non-cross-connected node which with two interfaces to connect its two neighbors, as well as a local interface to implement to increase or 
decrease the flow request. In this article, we only relate to cross-connect nodes, and the nodes connected to the cross SONET / WDM ring, which the two rings are interconnected between either a single node or multiple nodes.

As shown in Figure 1(a) and Figure 1(b):
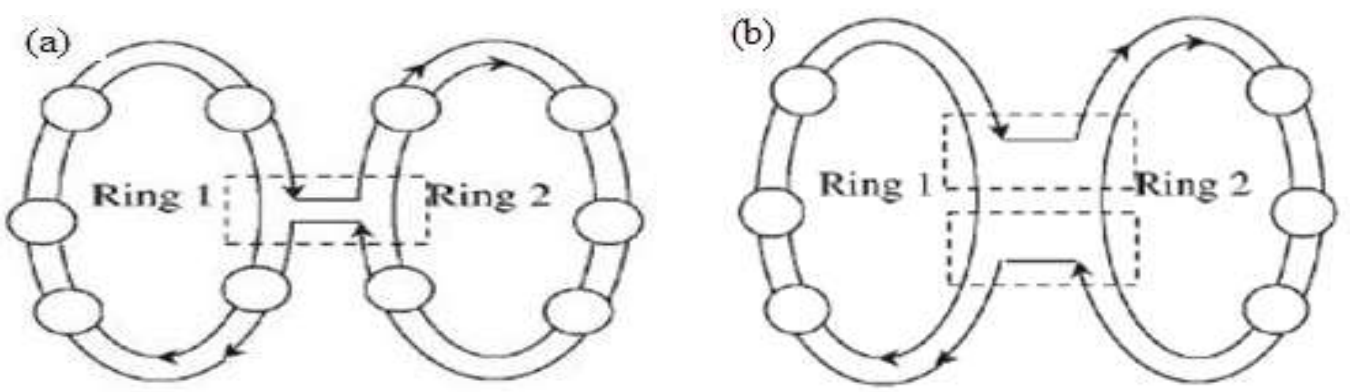

Figure 1 (a) Single -point ring connected, (b) Multi-point ring connected

If the traffic request go to another ring by optical switch, which we call a single jump. If the traffic request from one ring to reach a digital cross-connect (DXC) firstly, then reach another ring, which we call two-hop (or multiple jumps). This paper is based on the single-hop problem.

The literature has been given a ring network traffic grooming problem $(g>1)$ related concepts, and traffic grooming problem has proven to be NP-complete[3]. O. Gerstel, et al. who introduced the ring network, and minimized the number of the ADM in the case of $g=1$, and proved this problem is NP- complete [4] [5]. In a ring network, for any fixed value of g, M. Flammini etc. who have proposed an approximation algorithm with approximate ratio $2 \ln g+o(\ln g)$ [6]. In order to improve bandwidth utilization, B. Chen etc., Respectively, under different circumstances for the ring and star topology networks, traffic grooming technique using electronic processing equipment to minimize the problem of all-optical networks used in a profound study [ 7 ] [8] .

M.Flammini, etc. proposed an approximation algorithm in a tree network with approximate ratio $2 \ln g+\ln \Delta+o(\ln (\Delta \cdot g))$, which $\Delta$ is the largest degree of the nodes in the tree network [9].

\section{The definition of related issues}

Traffic grooming problem can be abstracted as a graph instance, which can be modeled as a graph coloring problem. An example of triplet $(\mathrm{G}, \mathrm{P}, \mathrm{g})$, Where $\mathrm{G}=(\mathrm{V}, \mathrm{E})$ is a request diagram, $\mathrm{P}$ is a simple path s set of $\mathrm{G}, \mathrm{g}$ is a positive integer, which called grooming factor.

Definition 2.1.1: Given a subset $Q \subseteq P$ and one edge e, $e \in E, Q_{e}$ is the paths set using edge e of Q. $l_{Q}(e)$ is the number of these paths, or with network jargon, which is the load on edge e which exported from $\mathrm{Q}$ path. $L_{Q}$ is the maximum load on any one edge of $\mathrm{G}$ which exported from path Q.

Definition 2.1.2 : A coloring(Wavelength assignment) of(G,P)is a function $\omega: P \mathrm{a} \mathrm{N}^{+}=\{1,2, \ldots\}$. We further extend the definition of the function $\omega$ on any subset $\mathrm{Q}$ of $\mathrm{P}$, $\omega(Q)=\mathrm{U}_{p \in Q} \omega(p)$. For a coloring $\omega$ 、 one color $\lambda$ and a subset $Q \subseteq P, Q_{\lambda}^{\omega}$ is colored $\lambda$ by $\omega$ in a path from a subset of Q.

Definition 2.1.3: A right coloring (Wavelength assignment) of $(G, P, g)$ is one coloring of $P$, for any one edge e, there are g paths with the same color coloring used edge e mostly. Formally, $\forall \lambda \in ¥^{+}, L_{P_{\lambda}^{\omega}} \leq g$.

Definition 2.1.4: If you use one color on the $\mathrm{Q}$ path coloring, then coloring $\omega$ is a 1-coloring of $Q \subseteq P$; if the presence of a right 1- coloring for path set $\mathrm{Q}$, then $\mathrm{Q}$ is1-colorable; if and only if $L_{Q} \leq g, Q \subseteq P$ is 1 -colorable. 
Definition 2.1.5: For one coloring $\omega_{\text {of }} \mathrm{P}$ and one node $v \in V, Q_{v}$ is a subset which has an endpoint in the set of paths Q of $v$. $v$ may be the source node or the destination node. Degree of a node $^{v}$ is the number of path which $v$ is the source node or destination node. In form, $d(v)=\left|Q_{v}\right|$; The degrees of the set of paths $\mathrm{Q}$ is the sum of the degree of all nodes in $\mathrm{Q} .{ }^{A D M_{\lambda}}$ is the number of ADM used in wavelength $\lambda$ on network. $A D M^{\omega}$ is the total number of ADM used in the network, that is, the total number of ADM on different wavelengths of operation.

In form,

$$
\begin{gathered}
d(Q)=\sum_{v} d(v)=\sum_{v}\left|Q_{v}\right| ; \\
A D M_{\lambda}^{\omega}(Q)=d\left(Q_{\lambda}^{\omega}\right) ; \\
A D M^{\omega}=\sum_{\lambda} A D M_{\lambda}^{\omega}(P)=\sum_{\lambda} d\left(P_{\lambda}^{\omega}\right)
\end{gathered}
$$

Traffic grooming problem is an optimization problem that to find a right coloring $\omega$ of instance $(G, P, g)$ such that the minimum number of ADM used in optical network.

\section{GROOMBYSC(k) algorithm}

Known an instance $(\mathrm{G}, \mathrm{P}, \mathrm{g})$ of traffic grooming problem, the algorithm has a factor $\mathrm{g}$ depends only on a parameter $\mathrm{k}$ of grooming factor $\mathrm{g}$, and the value of $\mathrm{k}$ is determined in the algorithm analysis.

The algorithm of GROOMBYSC(k) to achieve in three stages. The first stage, calculation 1colorable sets and their corresponding weights, considering the subset of paths $\mathrm{P}$ which size up to $k \cdot g$. When it finds a set of paths 1 - colorable Q when put Q and their corresponding weights added to the relevant set, which makes the path set $\mathrm{Q}$ ADM required minimum number ( when the path of Q colored with the same color ). The second phase, it is to find a set cover of path $\mathrm{P}$ with the subset calculated in the first stage, which using a minimum weight set cover problem proposed GREEDYSC approximation algorithm ${ }^{[10]}$ in this process. The third stage, by eliminating the set intersection, the set cover problem will be converted to a partitioning problem and then colored with the same color for each set of paths, each division are set to use a different color shading.

GROOMBYSC(k) algorithm is described as follows :

a)Phase 1- Prepare GREEDY input:

$S \leftarrow \phi$;

For each $U \subseteq V$, such that $|U| \leq k\{$

For every path set of every ring $Q \subseteq P_{U}$,

Such that $|Q| \leq k \cdot g\{$

If $Q$ is 1-colorabe , then \{

$S \leftarrow S \mathrm{U}\{Q\} ;$

Weight $[\mathrm{Q}]=\mathrm{d}(\mathrm{Q})$;

\}

\}

b)Phase 2-do GREEDYSC:

//Without loss of generality, set $S C=\left\{S_{1}, S_{2}, \ldots, S_{W}\right\}$

$S C \leftarrow G R E E D Y S C(S$, weight $)$.

c) Phase 3-Put set cover SC convert to a division PART: 


$$
P A R T \leftarrow \phi
$$

$$
\text { For } \mathrm{i}=1 \text { to } \mathrm{W}\left\{P A R T_{i} \leftarrow S_{i}\right\}
$$

As long as there are two disjoint sets $P A R T_{i}, P A R T_{j}\{$

$$
\begin{aligned}
& P A R T_{i} \leftarrow P A R T_{i} \backslash P A R T_{j} \\
& \text { For } \lambda=1 \text { to } \mathrm{W} \quad(\omega(Q)=W)\{ \\
& \quad P A R T \leftarrow P A R T \mathrm{U}\left\{P A R T_{\lambda}\right\} \\
& \text { For each } p \in P A R T_{\lambda} \quad\{\omega(p)=\lambda\}
\end{aligned}
$$

\section{Algorithm Analysis}

Correctness and run time

Correctness and time complexity of the algorithm is analyzed and has been given a similar $\operatorname{proof}^{[5]}$, Which has been demonstrated for any fixed value of $\mathrm{k}$ and the instance $(\mathrm{G}, \mathrm{P}, \mathrm{g})$, the run time of the algorithm is polynomial time in $n=|P|$ and $m=|E|$. We can prove to be applied by a similar cross-connect ring GROOMBYSC $(\mathrm{k})$ obtained by analyzing the algorithm, and its run time is polynomial time too in $n=|P|$ and $m=|E|^{[5]}$.

Approximation ratio

Lemma: Given an integer $t$, there is a solution $\overline{S C}$ for the instance on the set covering problem which determined in the second phase of GROOMBYSC (k) algorithm, we have $k=2 t-1$, such that

$$
\text { weight }(\overline{S C}) \leq A D M^{*}\left(1+\frac{2 g}{t}\right) \text {. }
$$

Proof: Set $\omega^{*}(P)=\left\{1,2, \ldots, W^{*}\right\}$ and $1 \leq \lambda \leq W^{*}$, considering the set $V_{\lambda}^{*}$ of node $\mathrm{v}$ such that $A D M_{\lambda}^{*}(v)=1$, that is on the node $\mathrm{v}$ has an operation wavelength $\lambda$ in the ADM. Start from any node in the clockwise direction of the ring will be set $V_{\lambda}^{*}$ into a plurality of at least $\mathrm{t}$ the set of nodes, and network nodes at most $k=2 t-1$ ( as shown in Figure 2). For cross two SONET rings connected, each ring have taken this division, the optimization problem are independent of each ring, reach ( or derived from ) the other traffic on the ring comes from ( or arrive ) cross node. Set $V_{\lambda, j}\left(j=1, \ldots, P_{\lambda}\right)$ is the subset of nodes in this way. Set $q_{\lambda}$ is the number of subset with at least $\mathrm{t}$ nodes in $V_{\lambda}^{*}$, if there has present the subset is less than $\mathrm{t}$ nodes in $V_{\lambda}^{*}$, then set $r_{\lambda} \leq t$ is the remaining number of nodes in the subset. In form, se $A D M_{\lambda}^{*}=\left|V_{\lambda}^{*}\right|=t q_{\lambda}+r_{\lambda}$, among them $r_{\lambda}=\left|V_{\lambda}^{*}\right| \bmod t$ and $0 \leq r_{\lambda} \leq t$.

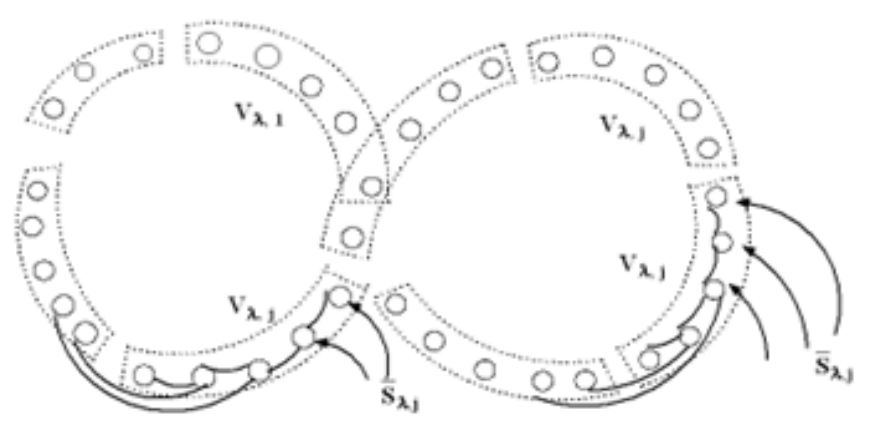

Figure 2: $\operatorname{Set} V_{\lambda, j}, \overline{S_{\lambda, j}}$ and $t=5$

Note that if no fewer than $\mathrm{t}$ nodes in the collection division, then $p_{\lambda}=q_{\lambda}$ and $r_{\lambda}=0$; Otherwise, $p_{\lambda}=q_{\lambda}+1$.Clearly, $\forall 1 \leq j \leq q_{\lambda},\left|V_{\lambda, j}\right|=t$ and in the case of $r_{\lambda}>0$, we have $\left|V_{\lambda, q_{\lambda}+1}\right|<t$; In both cases, we have $\left|V_{\lambda, j}\right| \leq t$, then $t \leq k$, we have $\left|V_{\lambda, j}\right| \leq k$. So $V_{\lambda, j}$ is added to the set $\mathrm{S}$. 
For $V_{\lambda, j}$, we define $\overline{S_{\lambda, j}}$ is the path set of $P_{\lambda}^{\omega^{*}}$, and there is counterclockwise endpoint in $V_{\lambda, j}$. Because $V_{\lambda, j}$ have t nodes at least, and each node may be a clockwise endpoint of at most $\mathrm{g}$ paths which is 1-colorable.Then, have $\left|\overline{S_{\lambda, j}}\right| \leq g \cdot k$. Therefore, $\overline{S_{\lambda, j}}$ is taken into account in the inner loop algorithm in the first stage. $\overline{S_{\lambda, j}}$ is a 1-colorabe,is added to set $\mathrm{S}$, that is, $\overline{S_{\lambda, j}} \in S$.

Two endpoints of each path ${ }^{p \in P_{\lambda}^{\omega^{*}}}$ are in the $\operatorname{set} V_{\lambda, j}$, specifically, for a certain value of $\mathrm{j}$, it has its endpoint in a clockwise in $V_{\lambda, j}$. So, it is an element of the set $\overline{S_{\lambda, j}}$, then we have $\overline{S C_{\lambda}}=\mathrm{U}_{j}\left\{S_{\lambda, j}\right\}$ is a set cover of $P_{\lambda}^{\omega^{*}}$. We conclude, for $1 \leq \lambda \leq W^{*}$, we have $\overline{S C}=\mathrm{U}_{\lambda=1}^{W^{*}} \overline{S C_{\lambda}}$ is a set cover of path set P. Let's prove that the weights have the following attributes:

We are known $A D M_{\lambda}^{*}=t q_{\lambda}+r_{\lambda}$, the sum of all possible values for $\lambda$, get $A D M^{*}=t \sum q_{\lambda}+\sum r_{\lambda}$, this means $\sum q_{\lambda} \leq \frac{A D M^{*}}{t}$. Two cases are considered:

(1) When $\forall j \leq q_{\lambda}$ weight $\left(\overline{S_{\lambda, j}}\right)=d\left(\overline{S_{\lambda, j}}\right) \leq t+g$.

Because:

a) The source and destination nodes are the endpoints of the paths $\overline{S_{\lambda, j}}$ are also in $V_{\lambda, j}$, and $\left|V_{\lambda, j}\right|=t$.

b) The source node and the destination node, only one is at most g paths in the path $V_{\lambda, j}$.

(2) When $j=q_{\lambda}+1$, weight $\left(\overline{S_{\lambda, j}}\right)=\leq g \cdot q_{\lambda}+r_{\lambda}$

Because:

a) Only one endpoint is at most $\mathrm{g}$ paths in $\mathrm{V}_{\lambda, \mathrm{q}_{\lambda}+1}$.

b) The source and destination nodes are the endpoints of the paths $\bar{S}_{\lambda, q \lambda+1}$ are also in $V_{\lambda, q_{\lambda}+1}$, and $\left|V_{\lambda, q_{\lambda}+1}\right|=r_{\lambda}$.

Summing all $1 \leq j \leq q_{\lambda}+1$, we have:

$$
\text { weight }\left(\overline{S C_{\lambda}}\right) \leq \sum_{j=1}^{q_{\lambda}}(t+g)+g \cdot q_{\lambda}+r_{\lambda}
$$

Because $A D M^{*}=t \sum_{\lambda} q_{\lambda}+\sum_{\lambda} r_{\lambda}$, and $\sum_{\lambda} q_{\lambda} \leq \frac{A D M^{*}}{t}$.

Thus, we get the following results:

$$
\begin{aligned}
\text { weight }(\overline{S C}) & =\sum_{\lambda} \text { weight }\left(\overline{S C_{\lambda}}\right) \leq A D M^{*}+2 g \sum_{\lambda} q_{\lambda} \\
& \leq A D M^{*}+2 g \cdot \frac{A D M^{*}}{t}=A D M^{*}\left(1+\frac{2 g}{t}\right)
\end{aligned}
$$

(2)Theorem: For bidirectional SONET ring in the cross-connect traffic grooming problem, there is an approximation algorithms with approximate ratio $2 \ln g+o(\ln g)$.

Proof: A greedy algorithm with minimize weight set covering problem is an $H_{f}$-approximation algorithm, among them, $f$ is the largest potential of algorithm input set and $H_{f}=1+1 / 2+\ldots+1 / f$ is the first number $f$ harmonic number.

We know $A D M^{\omega}=H_{f} \cdot$ weight $(\overline{S C})^{[5]}$, and weight $(\overline{S C}) \leq A D M^{*}\left(1+\frac{2 g}{t}\right)$,

So, we have:

$$
A D M^{\omega} \leq H_{f} \cdot A D M^{*}\left(1+\frac{2 g}{t}\right)
$$


Equivalent to

$$
\rho \leq H_{f} \cdot\left(1+\frac{2 g}{t}\right)
$$

We were carried out on the value of $t$ and $f$, take $t=g \ln g, f=(2 t-1) \cdot g$ (Only consider bidirectional SONET ring which has only one cross -way intersection connected), then,

$$
\begin{aligned}
\rho \leq & H_{(2 t-1) \cdot g} \cdot\left(1+\frac{2 g}{t}\right) \leq H_{(2 t-t) \cdot g} \cdot\left(1+\frac{2 g}{t}\right)=H_{t \cdot g} \cdot\left(1+\frac{2 g}{t}\right) \leq(1+\ln (t \cdot g))\left(1+\frac{2 g}{t}\right)=\left(1+\ln \left(g^{2} \ln g\right)\right)\left(1+\frac{2}{\ln g}\right) \\
= & (1+2 \ln g+\ln \ln g)\left(1+\frac{2}{\ln g}\right)=5+2 \ln g+\ln \ln g+\frac{2}{\ln g}+\frac{2 \ln \ln g}{\ln g}=2 \ln g+o(\ln g)
\end{aligned}
$$

Thus, the proof is completed.

\section{Conclusion}

This paper main highlights traffic grooming problem in cross-connect bidirectional SONET / WDM ring, and mainly studied the approximate ratio g of logarithmic approximation algorithm, and applied to solve the cross-connect bidirectional SONET / WDM ring traffic grooming problem, and through the analysis of the algorithm obtained approximate ratio $2 \ln g+o(\ln g)$. By proven processes and results, the approximation ratio of the algorithm is independent of the number of nodes in cross-connect bidirectional SONET/WDM ring, only depends on the grooming factor $\mathrm{g}$. Grooming factor smaller than its approximation ratio of the approximation algorithm is also smaller, the better the performance of the algorithm can be seen, which can greatly improve and optimize the network performance.

By the results of this verification in this article, GROOMBYSC $(\mathrm{k})$ approximation algorithm can be applied to any topology, through more accurate analysis and prove similar results can be obtained.

This article will be bidirectional SONET ring GROOMBYSC (k) approximation algorithm is applied in the cross-connect, get better performance, proved a general of the approximation algorithm.

\section{References}

[1] K.Zhu and B.Mukherjee. "A review of traffic grooming in wdm optical networks: Architecture and challenges." Optical Networks Magazine,4(2):55-64,March-April 2003.

[2] O.Gerstel, R.Ramaswami, and G..Sasaki. "Cost effective traffic grooming in wdm rings." In INFOCOM'98, Seventeenth Annual Joint Conference of the IEEE Computer and Communications Societies, 1998.

[3]Walter Goralski. Optical Networking \&WDM[M].Post \& Telecommunications Press.2003,pages 118-122,148-150.

[4] O. Gerstel, P. Lin, and G. Sasaki. "Wavelength assignment in a wdm ring to minimize cost of embedded sonet rings." In INFOCOM98, Seventeenth Annual Joint Conference of the IEEE Computer and Communications Societies, 1998.

[5] T. Eilam, S. Moran, and S. Zaks. "Lightpath arrangement in survivable rings to minimize the switching cost." IEEE Journal of Selected Area on Communications, 20(1):172 C 182, Jan 2002.

[6] M. Flammini, L. Moscardelli, M. Shalom, and S. Zaks. "Approximating the traffic grooming problem.” In ISAAC, pages 915 C 924, 2005. 
[7] B. Chen, G. N. Rouskas, and R. Dutta. "Traffic grooming in wdm ring networks with the min-max objective.” In NETWORKING, pages 174 C 185, 2004.

[8] B. Chen, G. N. Rouskas, and R. Dutta. "Traffic grooming in star networks.” In Broadnets, 2004.

[9] M. Flammini, G. Monaco, L. Moscardelli, M. Shalom, and S. Zaks, "Approximating the traffic grooming problem in tree and star networks," presented at the WG 2006 Workshop, Bergen, Norway, Mar. 2006.

[10] D. S. Johnson. "Approximation algorithms for combinatorial problems." J.Comput. System Sci., 9:256 C 278, 1974. 